In the Hestenes-Stiefel method, the corresponding count is $2 n^{2}+5 n+4$. In the latter method, if $\rho_{n}$ in the last step were to be computed from the recursion relation, the method would win back the $n^{2}$ multiplications which it lost to the Craig method in the first step; but it would hardly be reasonable to calculate the last residual in this way.

We note that since the algorithm in either case will very probably last for $n$ steps unless a peculiarly fortunate choice of $\xi_{0}$ was made, the total number of multiplications to be expected is of the order of $2 n^{3}$. The eventual justification for the use of conjugate gradient methods in preference to the most economical direct methods (which theoretically require only $n^{3} / 3+$ $O\left(n^{2}\right)$ multiplications) must rest on definitive $a$ priori studies of round-off error, and on the peculiarities of individual computing machines, and on special characteristics of the matrix $A$. But it seems quite probable at this writing that no final recommendations will ever be formulated as to the "best" ways of solving linear equations.

There is a further practical use for the algorithm $(1)^{\prime}-(5)^{\prime}$ other than the applications represented by the specializations described above. It may be possible under some circumstances to find a matrix $B$ such that premultiplication of the equation $A \xi=\eta$ by $B$ will improve the "condition"'s of $A$. Then $(1)^{\prime}-(5)^{\prime}$, with $T$ chosen conveniently, say $T=\left(A^{\prime} A\right)^{-1}$, might give an advantageous algorithm to use. (With $T=\left(A^{\prime} A\right)^{-1}$ the algorithm would be the same as that of Case 2 but with $A$ replaced by $B^{\prime}$.)

In conclusion, we note that Hestenes \& Stiefel $^{1}$ assert (p. 424-425) that the most general cg-algorithm for any linear system is obtained from (1)'$(5)^{\prime}$ by assuming that $B A$ is positive definite and $T=\left(A^{\prime} B^{\prime}\right)^{-1}$. Nevertheless without this specialization, the generality of $(1)^{\prime}-(5)^{\prime}$ seems to exceed the limits specified by Hestenes \& Stiefel, because here $B$ is an arbitrary non-singular matrix. The disagreement, if it may be called that, seems to lie in the fact that their characterization of generality is derived from a certain generalization of the method of conjugate directions, and this generalization does not include all the possibilities represented by the algorithm given here.

New York Univ.

J. H. CuRTISS

New York, N. Y.

${ }^{1}$ M. R. Hestenes \& E. Stiefel, "Methods of conjugate gradients for solving linear systems," NBS Jn. of Research, v. 49, 1952, p. 409-436.

2 E. J. CRAIG, Iteration Procedures for Simultaneous Equations. Doctoral dissertation, Mass. Inst. Tech., Cambridge, Mass., 1954.

${ }^{3}$ J. ToDD, "The condition of certain matrices, I," Quart. Jn. of Mech. and Appl. Math., v. 2,1949 , p. $469-472$.

\title{
On the Estimation of Quadrature Errors for Analytic Functions
}

1. Introduction. The present paper sets forth a complex variable method for the estimation of errors which arise when approximate rules of quadrature are applied to analytic functions. In contrast to the usual real variable methods, this method does not involve the use of the higher derivatives of the function, but uses only a knowledge of the size of the function in the complex plane. It is therefore of practical value when dealing with situations 
in which the higher derivatives of the function are difficult to obtain in closed form, or where the higher derivatives become excessively large over the interval in question.

The principal feature of this method is the use of the characteristic inequality for bounded linear functionals. The method was developed substantially in DAvis ${ }^{2}$ and outlined there not only for quadratures but also for rules of differentiation, interpolation, etc. Numerical coefficients were obtained for several rules for the case of functions analytic in the unit circle. In the present paper, we consider only quadrature formulas. We deal with certain commonly applied rules of low order and also some Gaussian rules of rather high order, and present a table of coefficients (Table 2) which permits the rapid estimation of errors (through formulas (26), (31), (38)) when these rules are applied to functions which are analytic on a closed interval $[a, b]$. The estimates given here provide upper bounds for the error and their use leads to a conservative practice. In a certain sense, as will be explained in $\S 4$, these estimates are best possible. The problem of obtaining very precise estimates of error is a difficult one and such estimates can be obtained only after a painstaking analysis of the particular function integrated. When estimates are developed which are applicable to wide classes of functions, some precision is necessarily lost. It is felt that the present method provides a compromise between the time involved in making the estimate and the precision of the estimate. It should be added that in any case, the precision of the present estimates is independent of the precision of conventional estimates obtained by using the maximum value of some high derivative over the integration interval; that is, for some functions the former will provide the sharper estimate while for other functions the latter will.

The consideration of classes of functions which are analytic on a closed interval (as opposed to functions which are analytic in a fixed circle) provides more freedom with but slightly more work. This freedom shows up particularly when dealing with functions which are analytic on the segment of integration but which have singularities within the circle constructed on this segment as a diameter. Functions which are analytic on the interval of integration are most conveniently handled by the introduction of the Chebyshev polynomials. These polynomials are orthogonal (with respect to a weight) not only over the real interval but over certain ellipses in the complex plane, and the resulting complex analytic Fourier analysis lends considerable simplicity to the developments which follow.

We reiterate that we are dealing with functions which are analytic on a closed line segment and hence are continuable analytically to some two dimensional region of the complex plane which contains that segment in its interior. Thus, we rule out the cases where the function to be integrated has only low order continuity properties or the case where the function has been extracted from an ensemble of functions with only certain statistical facts known.

2. The Class $L^{2}\left(\boldsymbol{\varepsilon}_{p}\right)$. Let $B$ designate a bounded region lying in the plane of the complex variable $z=x+i y$. By $L^{2}(B)$, we shall mean the class of functions which are single-valued regular analytic in $B$ and are such that

$$
\iint_{B}|f(z)|^{2} d x d y<\infty .
$$


The class $L^{2}(B)$ has been studied extensively. For the fundamentals of this theory see BERGMAN. ${ }^{1}$ We note in particular that if $f(z)$ is regular in the closure of $B$, then (1) holds. The positive square root of the quantity (1) is generally termed the norm of $f$ over $L^{2}(B)$ and is designated by $\|f\|$ or $\|f\|_{B}$. That is,

$$
\|f\|_{B}=\left(\iint_{B}|f(z)|^{2} d x d y\right)^{\frac{1}{2}} ; \quad f \in L^{2}(B) .
$$

If a region $G$ satisfies $G \subseteq B$ and if $f \in L^{2}(B)$, it is clear that $f \in L^{2}(G)$ and that

$$
\|f\|_{G} \leq\|f\|_{B} .
$$

The principal features of the class $L^{2}(B)$ are as follows. We introduce the integral

$$
\iint_{B} f(z) \overline{g(z)} d x d y=(f, g) ; \quad f, g \in L^{2}(B),
$$

as an inner product. The class $L^{2}(B)$ possesses complete orthonormal systems $\left\{\zeta_{n}(z)\right\}$ in the sense of (4). Every function of the class can be expanded in a Fourier series

$$
f(z)=\sum_{n=0}^{\infty} a_{n} \zeta_{n}(z)
$$

wherein

$$
a_{n}=\left(f, \zeta_{n}\right) \quad(n=0,1, \cdots) .
$$

The convergence of $(5)$ is uniform and absolute in every closed subregion of $B$. Moreover, we have

$$
\|f\|^{2}=\sum_{n=0}^{\infty}\left|a_{n}\right|^{2}
$$

The bilinear series $\sum_{n=0}^{\infty} \zeta_{n}(z) \overline{\zeta_{n}(w)}$ converges for $z$, $w \in B$ to a function $K_{B}(z, \bar{w})$, generally designated as the Bergman kernel function of $B$, which posseses the characteristic reproducing property, $\left(f(z), K_{B}(z, \bar{w})\right)=f(w)$ for all $f \in L^{2}(B)$. For simply connected regions $B$ with a Jordan boundary, there exists a complete orthonormal set of polynomials $\left\{p_{n}(z)\right\}$.

There are, moreover, two notable cases wherein the polynomials $p_{n}(z)$ have a particularly simple structure. They are the circle and the ellipse. The circle $|z|<R$ possesses the set of polynomials

$$
p_{n}(z)=(n+1)^{\frac{1}{2}} \pi^{-\frac{1}{2}} z^{n} R^{-n-1} \quad(n=0,1, \cdots)
$$

as a complete orthonormal system.

To discuss the case of the ellipse, it is best to assume that it has been placed in a normalized position. Let an ellipse, therefore, have its major axis along the $x$ axis and its foci at the points $(-1,0)$ and $(1,0)$. Let $a$ and $b=$ $\left(a^{2}-1\right)^{\frac{1}{2}}$ designate its semimajor and semiminor axes respectively and let 
the quantity $\rho=\rho(a)$ be defined by

$$
\rho=(a+b)^{2}, \quad a=\frac{1}{2}\left(\rho^{\frac{1}{2}}+\rho^{-\frac{1}{2}}\right), \quad b=\frac{1}{2}\left(\rho^{\frac{1}{2}}-\rho^{-\frac{1}{2}}\right) .
$$

This ellipse will be designated by $\mathcal{E}_{\rho}$. For values of $\rho>1$, these ellipses form a confocal family and collapse to the segment $[-1,+1]$ as $\rho \rightarrow 1$. In Table 1 will be found the values of the geometric quantities $b$ and $\rho$ for a number of selected values of $a$.

We introduce the Chebyshev polynomials of the second kind by means of the definition

$$
U_{n}(z)=\left(1-z^{2}\right)^{-\frac{1}{2}} \sin ((n+1) \arccos z) \quad(n=0,1, \cdots) .
$$

It can then be shown that the polynomials

$$
\zeta_{n}(z)=2(n+1)^{\frac{1}{2}} \pi^{-\frac{1}{2}}\left(\rho^{n+1}-\rho^{-n-1}\right)^{-\frac{1}{2}} U_{n}(z), \quad(n=0,1, \cdots)
$$

form a complete orthonormal system for $L^{2}\left(\mathcal{E}_{\rho}\right)$.

3. Quadrature Errors for $L^{2}\left(\varepsilon_{\rho}\right)$. In conformity with the above normalization, we shall assume that the integration to be performed is over the interval $[-1,1]$. The case of an arbitrary interval may be handled by means of an appropriate linear transformation [see §6]. An arbitrary $N+1$ point quadrature formula is given by

$$
\int_{-1}^{+1} f(x) d x \sim \sum_{k=0}^{N} a_{k} f\left(\lambda_{k}\right) \equiv R(f)
$$

where $\lambda_{k}$ are certain abscissas lying in $[-1,1]$ and $a_{k}$ are the associated weights. The error $E \equiv E(f)$ involved in the rule $R$ is

$$
E(f)=\int_{-1}^{1} f(x) d x-R(f)
$$

and may be regarded as a linear functional on $f$.

If $f(x)$ is analytic on $[-1,1]$ then it is clear that for some value of $\rho>1$, $f$ may be continued analytically so as to be regular in the closed ellipse $\overline{\mathcal{E}}_{\rho}$. Such an $f(z)$ is therefore of class $L^{2}\left(\mathcal{E}_{\rho}\right)$, and may be expanded in a series of Chebyshev polynomials,

$$
f(z)=\sum_{n=0}^{\infty} a_{n} \zeta_{n}(z) ; \quad \sum_{n=0}^{\infty}\left|a_{n}\right|^{2}=\|f\|^{2}<\infty,
$$

which converges uniformly and absolutely in the interior of $\mathcal{E}_{\rho}$. Applying the operator $E$ to (14) we obtain

$$
E(f)=\sum_{n=0}^{\infty} a_{n} E\left(\zeta_{n}\right) .
$$

Estimating $E(f)$ by means of the Schwarz inequality, there is obtained

$$
|E(f)|^{2} \leq \sum_{n=0}^{\infty}\left|a_{n}\right|^{2} \sum_{n=0}^{\infty}\left|E\left(\zeta_{n}\right)\right|^{2} .
$$


Let us now write

$$
\sigma^{2}=\sigma_{R}^{2}=\sum_{n=0}^{\infty}\left|E\left(\zeta_{n}\right)\right|^{2}
$$

then in view of $(7)$ there is obtained

$$
|E(f)| \leq \sigma_{R}\|f\| \text {. }
$$

The quantity $\sigma_{R}$ which is the norm over $L^{2}\left(\mathcal{E}_{\rho}\right)$ of the bounded linear functional $E$ has an alternate representation in the form $\sigma_{R}{ }^{2}=E_{z} E_{\bar{w}} K_{B}(z, \bar{w})$. It therefore depends only upon the ellipse $\mathcal{E}_{\rho}$, and the quadrature rule $R$, but is independent of $f$ and of the particular complete orthonormal set of functions used in the expansion (14). The quantity $\sigma_{R}$ may therefore be computed once for all. Using (17) and (11) we have

$$
\sigma_{R}^{2}=\frac{4}{\pi} \sum_{n=0}^{\infty}(n+1) \frac{\left|E\left(U_{n}(z)\right)\right|^{2}}{\rho^{n+1}-\rho^{-n-1}} .
$$

We have, moreover,

$$
U_{n}(z)=(n+1)^{-1} T^{\prime}{ }_{n+1}(z) \quad(n=0,1, \cdots),
$$

where $T_{n}(z)$ designates the Chebyshev polynomials of the first kind defined by

$$
T_{n}(z)=\cos (n \arccos z) \quad(n=0,1, \cdots) .
$$

Therefore,

$$
\begin{aligned}
\int_{-1}^{+1} U_{n}(x) d x=\frac{1}{n+1}\left[T_{n+1}(1)\right. & \\
& \left.-T_{n+1}(-1)\right]=\frac{1}{n+1}\left[1+(-1)^{n}\right] .
\end{aligned}
$$

If quantities $\tau_{n}$ are defined by

then we have

$$
\begin{array}{ll}
\tau_{n}=0 & n \text { odd } \\
\tau_{n}=\frac{2}{n+1} & n \text { even, }
\end{array}
$$

$$
\sigma_{R}^{2}=\frac{4}{\pi} \sum_{n=0}^{\infty} \frac{(n+1)}{\rho^{n+1}-\rho^{-n-1}}\left(\tau_{n}-\sum_{k=0}^{N} a_{k} U_{n}\left(\lambda_{k}\right)\right)^{2} .
$$

Table 2 lists the values of $\sigma$ corresponding to the trapezoidal, Simpson's, Weddle's and the Gaussian 2,3,7,10 and 16-point formulas [For the abscissas and weights of these Gaussian rules see LowAN $\left.{ }^{4}\right]$, and for a range of values of the parameter $\rho$. These values were computed from (24) on the National Bureau of Standards Eastern Automatic Computer (SEAC).

4. On the Error Estimate (18). The inequality (18) allows us to estimate quadrature errors from a table of values of $\sigma$ and from an estimate of the norm $\|f\|$. Let us assume that we are dealing with a fixed rule $R$ with error $E$. The value of the parameter $\rho$ is at our disposal to a certain extent. For func- 
tions $f(x)$ which are analytic on $[-1,1]$ there will be a range of values of $\rho$, $1<\rho<\rho^{*} \leq \infty$ for which $f(z) \epsilon L^{2}\left(\mathcal{E}_{\rho}\right)$. For each such $\rho$, the inequality (18) is valid. To exhibit the dependence of (18) upon $\rho$, we should write

$$
|E(f)| \leq \sigma_{R}(\rho)\|f\|_{\varepsilon_{\rho}}, \quad 1<\rho<\rho^{*} .
$$

Now $\|f\|_{\varepsilon_{\rho}}=0$ when $\rho=1$ and increases as $\rho$ increases. On the other hand $\sigma_{R}(\rho)$ decreases as $\rho$ increases. Hence, the best estimate will occur for some intermediate value of $\rho$. The inequality $\left(18^{\prime}\right)$ can therefore be improved by writing

$$
|E(f)| \leq \min _{1<\rho<\rho^{*}} \sigma_{R}(\rho)\|f\|_{\varepsilon_{\rho}}
$$

Let the rule $R$ as well as the ellipse $\mathcal{E}_{\rho}$ be fixed. Then the inequality (18') (which is the characteristic inequality for bounded linear functionals) is a best possible one in the following sense : there exist functions $g$ of class $L^{2}\left(\mathcal{E}_{\rho}\right)$ such that

$$
|E(g)|=\sigma_{R}(\rho)\|g\|_{\varepsilon_{\rho}} .
$$

In general, of course, the error will be less. There are two additional facts which in the practical carrying out of this method tend to diminish the precision of the method. The first is that the exact minimum in (25) is difficult to ascertain and what one does is simply to take the minimum of a finite number of such values. The second is that the norm of $f$ cannot be computed exactly except in the simplest cases and an appropriate upper bound must be used. The net effect of all this is to replace the right hand side of (25) with a less precise upper bound, but one which is much more readily ascertained.

5. Methods for Estimating $\|f\|$. It appears, then, that the principal task confronting the numerical analyst when using the present method is that of estimating the norm $\|f\|$ over some ellipse $\mathcal{E}_{\rho}$. In the present section we shall explain a number of devices which may be useful for this purpose. By the very definition (2) we have

$$
\|f\|_{\varepsilon_{\rho}}^{2}=\iint_{\varepsilon_{\rho}}|f(z)|^{2} d x d y,
$$

and it may be possible in certain simple cases to evaluate (27) directly. If $f(z)$ is continuous in the closed ellipse $\overline{\mathcal{E}}_{\rho}$, and if we set

$$
M_{\rho}=\max _{z \in \tilde{\delta}_{\rho}}|f(z)|,
$$

then we have from (27),

$$
\|f\|_{\varepsilon_{\rho}}{ }^{2} \leq M_{\rho}^{2} \iint_{\varepsilon_{\rho}} d x d y,
$$

so that

$$
\|f\|_{\varepsilon_{\rho}} \leq \sqrt{\pi a b} M_{\rho}
$$


where the quantities $a$ and $b$ are related to $\rho$ by means of (9). The quantity $M_{\rho}$ or an upper bound for it can, in many cases, be obtained by algebraic manipulation. It may be more convenient to replace $\mathcal{E}_{\rho}$ by some circle containing it, say the circle $C_{a}$ :

We then have

$$
|z| \leq a=\frac{\rho+1}{2 \rho^{\frac{3}{2}}} .
$$

$$
\|f\|_{\varepsilon_{\rho}}<\|f\|_{c_{a}} \leq a \sqrt{\pi} \max _{|z| \leq a}|f(z)|,
$$

assuming that $f(z)$ is regular in $\bar{C}_{a}$.

If $f(z)$ is of class $L^{2}\left(C_{a}\right)$ and its Taylor expansion is known:

$$
f(z)=\sum_{n=0}^{\infty} \alpha_{n} z^{n}
$$

then it follows from (7) and (8) that we have

$$
\|f\|^{2} c_{a}=\pi a^{2} \sum_{n=0}^{\infty} \frac{\alpha_{n}^{2} a^{2 n}}{n+1}
$$

which is an exact evaluation of the middle term of the inequality (31). The quantity $\|f\|_{\delta_{\rho}}$ may itself be expressed as an infinite series involving the Taylor coefficients $\alpha_{n}$, but such expressions are more cumbersome and consequently less useful.

It should be pointed out that in cases of great complexity, there is always the possibility of obtaining $M_{\rho}$ directly by evaluating $|f(z)|$ along the boundary of $\mathcal{E}_{\rho}$ on a sufficiently dense set of points. Despite the loss of accuracy involved in combining (18) and (30), errors obtained in this way will, in general, be better than those obtained by using the conventional real variable error expressions and estimating the derivatives which occur there by means of Cauchy's inequality. This can be explained by the fact that the real variable expressions must be valid for a wider class of functions.

6. The Case of an Arbitrary Interval. The quantities of Tables 1 and 2 refer to the interval $[-1,1]$. The case of an arbitrary interval $[a, b]$ is dealt with by means of the linear transformation

$$
\left\{\begin{array}{l}
x=\frac{2 w}{b-a}-\frac{b+a}{b-a} \\
w=\frac{b-a}{2} x+\frac{b+a}{2}
\end{array}\right.
$$

which carries the interval $a \leq w \leq b$ into $-1 \leq x \leq 1$. Let the rule $E^{*}$ be given on $[a, b]$ as follows:

$$
E^{*}(f)=\int_{a}^{b} f(w) d w-\sum_{k=0}^{N} a_{k} f\left(\lambda_{k}\right) .
$$

The analogous rule on $[-1,1]$ is given by

$$
E(f)=\int_{-1}^{+1} f(x) d x-\sum_{k=0}^{N}\left(\frac{2}{b-a}\right) a_{k} f\left[\left(\frac{2 \lambda_{k}}{b-a}\right)-\left(\frac{b+a}{b-a}\right)\right]
$$


TABLE I : Geometric Quantities for Ellipses

$\begin{array}{cccc}a & b & \rho & (\pi a b)^{\frac{1}{3}} \\ 1.01 & .1418 & 1.3266 & .6607 \\ 1.02 & .2010 & 1.4908 & .8026 \\ 1.03 & .2468 & 1.6302 & .8936 \\ 1.04 & .2857 & 1.7574 & .9661 \\ 1.05 & .3202 & 1.8773 & 1.0277 \\ 1.10 & .4583 & 2.4282 & 1.2584 \\ 1.15 & .5679 & 2.9511 & 1.4324 \\ 1.20 & .6633 & 3.4720 & 1.5814 \\ 1.25 & .7500 & 4.0000 & 1.7162 \\ 1.30 & .8307 & 4.5397 & 1.8420 \\ 1.40 & .9798 & 5.6634 & 2.0759 \\ 1.50 & 1.1180 & 6.8541 & 2.2953 \\ 1.75 & 1.4361 & 10.1515 & 2.8099 \\ 2.00 & 1.7321 & 13.9282 & 3.2989 \\ 2.50 & 2.2913 & 22.9565 & 4.2421 \\ 3.00 & 2.8284 & 33.9706 & 5.1631 \\ 4.00 & 3.8730 & 61.9839 & 6.9763 \\ 5.00 & 4.8990 & 97.9898 & 8.7723\end{array}$

and is known by the same name. If $f(w)$ is analytic on $[a, b]$, then

$$
g(x)=f\left[\left(\frac{b-a}{2}\right) x+\frac{b+a}{2}\right]
$$

is analytic on $[-1,1]$ and, setting $x_{k}=\frac{2 \lambda_{k}}{b-a}-\frac{b+a}{b-a}$, we have

$$
\begin{aligned}
E^{*}(f)=\int_{a}^{b} f(w) d w-\sum_{k=0}^{N} a_{k} f\left(\lambda_{k}\right)=\frac{b-a}{2} & {\left[\int_{-1}^{+1} g(x) d x\right.} \\
& \left.-\sum_{k=0}^{N} a_{k} g\left(x_{k}\right)\left(\frac{2}{b-a}\right)\right],
\end{aligned}
$$

so that from (36), (35), and (34),

$$
E^{*}(f)=\left(\frac{b-a}{2}\right) E(g)
$$

Thus,

$$
\left|E^{*}(f)\right|=\frac{b-a}{2}|E(g)| \leq\left(\frac{b-a}{2}\right) \sigma_{R}(\rho)\|g\|_{\varepsilon_{\rho}} .
$$

The $\sigma_{R}(\rho)$ are the tabulated values [Table 2] in the $z=x+i y$ plane and $\|g\|_{\varepsilon_{\rho}}$ also refers to this plane.

\section{Examples.}

1. Estimate the error $E$ incurred in evaluating $\int_{0}^{1} \exp \left(e^{x}\right) d x$ by Weddle's rule. From (35) we have $g(z)=\exp \left(e^{(z+1) / 2}\right)$ which is an entire 


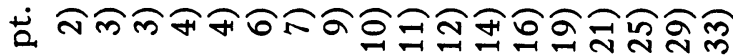

- I I I I I I I I I I I I I I I 1

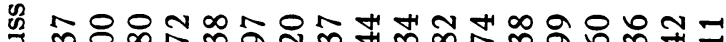

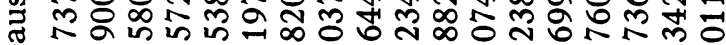

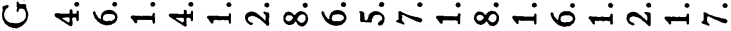

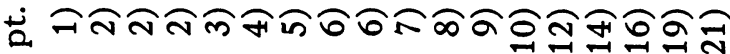

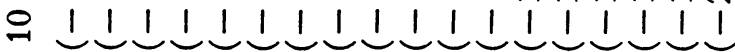

\% 숭요

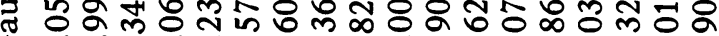

U i in $\mathrm{i}$ -

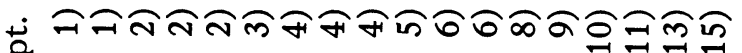

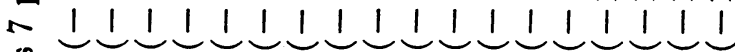

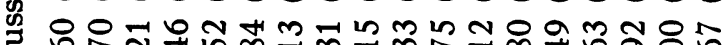

๘

+i

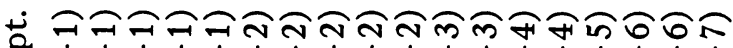

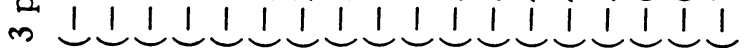

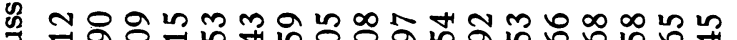

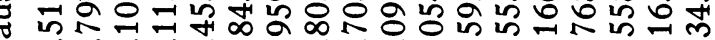

()

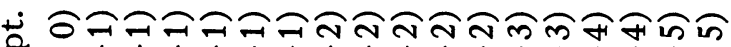

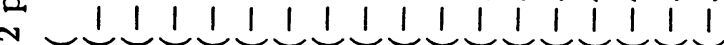

娄 $\infty=m a-1=m$ ก

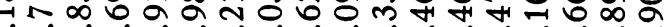

U -

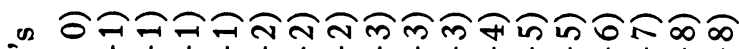

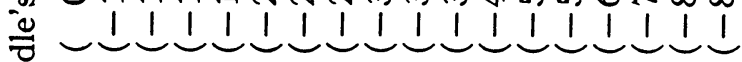

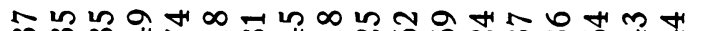

బึ)

-

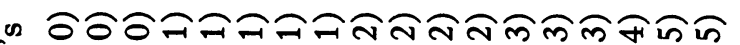

हี

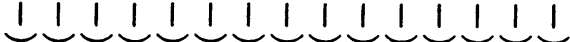

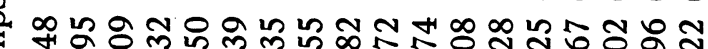

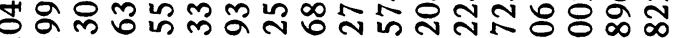
+i -iån

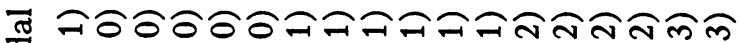

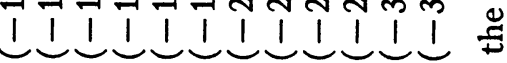

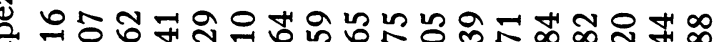

స่ 8 유ำ

H

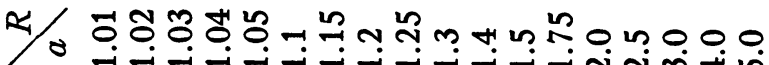


function of $z$ and is therefore of class $L^{2}\left(\mathcal{E}_{\rho}\right)$ for all $\rho>1$. Now, $\left|\exp \left(e^{(z+1) / 2}\right)\right|$ $=\exp \left[\operatorname{Re}\left(e^{(z+1) / 2}\right)\right]=\exp \left[e^{(x+1) \cdot 2} \cos y / 2\right]$. Thus on $\mathcal{E}_{\rho}$ we have $\mid \exp$ $\left(e^{(z+1) / 2}\right) \mid \leq \exp \left[e^{(a+1) / 2}\right]$. By (38) and (30) we have

$$
|E|<\frac{1}{2}(\pi a b)^{\frac{1}{2}} \exp \left[e^{(a+1) / 2}\right] \sigma_{w} .
$$

According to $\left(18^{\prime}\right)$, the estimate (39) is valid for all $a>1$. Of the values tabulated, the right member of (39) is minimized for $a=2.5$ and yields

$$
|E|<\frac{1}{2}(4.242)(315.7)\left(2.836 \times 10^{-6}\right) \doteq .0019 \text {. }
$$

In the above work, the norm of $\exp \left(e^{(z+1) / 2}\right)$ has been estimated crudely, and a number of improvements suggest themselves. Thus, for instance, | exp $\left(e^{(z+1) / 2}\right) \mid<\exp \left(e^{(x+1) / 2}\right)$, and since this last function is concave upward, we have

$$
\begin{array}{r}
\exp \left(e^{(x+1) / 2}\right) \leq \frac{1}{2}\left[\exp \left(e^{(a+1) / 2}\right)+\exp \left(e^{(-a+1) / 2}\right)\right]+\frac{x}{2 a}\left[\exp e^{(a+1) / 2}\right) \\
\left.-\exp \left(e^{(-a+1) / 2}\right)\right],-a \leq x \leq a .
\end{array}
$$

It is now easily verified that for arbitrary $A, B, C$,

$$
\iint_{\varepsilon_{\rho}}(A x+B y+C)^{2} d x d y=\pi a b\left(\frac{A^{2} a^{2}}{4}+\frac{B^{2} b^{2}}{4}+C^{2}\right),
$$

so that we have

where

$$
|E|<\frac{1}{2}(\pi a b)^{\frac{3}{3}}\left(\frac{A^{2} a^{2}}{4}+C^{2}\right)^{3} \sigma_{w}
$$

$$
\begin{aligned}
A & =\frac{1}{2 a}\left[\exp \left(e^{(a+1) / 2}\right)-\exp \left(e^{(-a+1) / 2}\right)\right], \\
C & =\frac{1}{2}\left[\exp \left(e^{(a+1) / 2}+\exp \left(e^{(-a+1) / 2}\right)\right] .\right.
\end{aligned}
$$

For $a=2.5$, we have $A=162.82, C=158.65$, so that

$$
|E|<\frac{1}{2}(4.2421)(160.6)\left(2.836 \times 10^{-6}\right)=.00097
$$

A conventional estimate of error using the formula

$$
|E| \leq \max _{0 \leq \xi \leq 1}\left[\frac{h^{7}}{140} f^{(6)}(\xi)+\frac{9 h^{9}}{1400} f^{(8)}(\xi)\right] ; f(x)=\exp \left(e^{x}\right),
$$

yielded the value $|E| \leq .006$.

2. Estimate the error $E$ incurred in evaluating $\int_{3}^{4} \Gamma(w) d w$ by means of the 7-point Gaussian rule. This is a case where conventional methods cannot be used due to the lack of information about the higher derivatives of the integrand. Transferring to the interval $[-1,1]$ we must consider the function $g(z)=\Gamma\left(\frac{1}{2}(z+7)\right)$. This function is regular in $|z|<7$; hence we may take $a$ in the range $1<a<7$. Now, $|\Gamma(x+i y)| \leq \Gamma(x)$ for $x>0$ 
so that $|g(z)|=\left|\Gamma\left(\frac{1}{2}(x+7)+\frac{1}{2} i y\right)\right| \leq \Gamma\left[\frac{1}{2}(x+7)\right]$. By the concavity of the $\Gamma$ function, $|g(z)| \leq \max \left\{\Gamma\left(\frac{1}{2}(a+7)\right), \Gamma\left(\frac{1}{2}(-a+7)\right)\right\}, z \in \mathcal{E}_{\rho}$. Thus we have from (38) and (30),

$$
|E|<\frac{1}{2}(\pi a b)^{\frac{1}{2}} \max \left\{\Gamma\left(\frac{1}{2}(a+7)\right), \Gamma\left(\frac{1}{2}(-a+7)\right)\right\} \sigma_{G_{7}(a)} .
$$

The selection $a=5.0$ yields

$$
\begin{aligned}
|E| \leq \frac{1}{2}(8.772)(120)\left(3.867 \times 10^{-15}\right) \doteq 2.04 & \times 10^{-12} . \\
& \text { P. DAVIS } \\
& \text { P. RABINOWITZ }
\end{aligned}
$$

NBS

${ }^{1}$ S. Bergman, Kernel Functions and Conformal Mapping, Math. Surveys No. 5, New York, 1950

2'P. DAvis, "Errors of numerical approximation for analytic functions," $J n$. of Rational Mech. and Analysis, v. 2, 1953, p. 303-313.

${ }^{3}$ C. Lanczos, Introduction, Tables of Chebyshev Polynomials. NBS Applied Math., Ser. 9, Washington, D. C., 1952.

${ }^{4}$ A. N. Lowan, Norman Davids \& Arthur Levinson, "Table of the zeros of the Legendre polynomials of order 1-16 and the weight coefficients for Gauss' mechanical quadrature formula," Am. Math. Soc., Bull., v. 48, 1942, p. 739-743. formula.

${ }^{5}$ See, e.g., W. Magnus \& F. Oberhetringer, Formeln und Sätze, 2nd ed., p. 3, 1st

\section{Integrals Occurring in Problems of Molecular Structure}

In theoretical work on molecular structure based either upon the valence bond method or upon the molecular orbital method, the electronic wave functions are usually built up from atomic orbitals (AO's). The calculation of most physical and chemical quantities then reduces to the evaluation of a number of integrals involving these orbitals. It is customary to adopt SLATER $^{1}$ type AO's, defined by (cf. RüDENBERG ${ }^{2}$ )

$$
\begin{aligned}
(1 s) & =\left(\zeta^{3} / \pi\right)^{\frac{1}{3}} e^{-\zeta r} & (3 d \bar{\pi}) & =\left(2 \zeta^{7} / 3 \pi\right)^{\frac{1}{3}} y z e^{-\zeta r} \\
(2 s) & =\left(\zeta^{5} / \pi\right)^{\frac{1}{2}} r e^{-\zeta r} & (3 s) & =\left(2 \zeta^{7} / 5 \pi\right)^{\frac{1}{3}}\left(r^{2} / 3\right)^{-\zeta r} \\
(2 p \sigma) & =\left(\zeta^{5} / \pi\right)^{\frac{1}{2}} z e^{-\zeta r} & (3 p \sigma) & =\left(2 \zeta^{7} / 15 \pi\right)^{\frac{1}{3}} z r e^{-\zeta r} \\
(2 p \pi) & =\left(\zeta^{5} / \pi\right)^{\frac{1}{2}} x e^{-\zeta r} & (3 p \pi) & =\left(2 \zeta^{7} / 15 \pi\right)^{\frac{1}{3}} x r e^{-\zeta r} \\
(2 p \bar{\pi}) & =\left(\zeta^{5} / \pi\right)^{\frac{1}{3}} y e^{-\zeta r} & (3 p \bar{\pi}) & =\left(2 \zeta^{7} / 15 \pi\right)^{\frac{1}{3}} y r e^{-\zeta r} \\
(3 d \sigma) & =\left(\zeta^{7} / 2 \pi\right)^{\frac{1}{3}}\left(z^{2}-r^{2} / 3\right) e^{-\zeta r} & (3 d \delta) & =\left(2 \zeta^{7} / 3 \pi\right)^{\frac{1}{3}} \frac{1}{2}\left(x^{2}-y^{2}\right) e^{-\zeta r} \\
(3 d \pi) & =\left(2 \zeta^{7} / 3 \pi\right)^{\frac{1}{3}} x z e^{-\zeta r} & (3 d \bar{\delta}) & =\left(2 \zeta^{7} / 3 \pi\right)^{\frac{1}{3}} x y e^{-\zeta r}
\end{aligned}
$$

where $(x, y, z)$ are the cartesian coordinates of the electron referred to the nucleus as origin with the $z$-axis directed along the internuclear axis towards the other nucleus, $r^{2}=x^{2}+y^{2}+z^{2}$ and $\zeta$ is a numerical screening parameter. Occasionally complex Slater type AO's are used; however since these are simply linear combinations of the real Slater AO's, no distinction will be made between integrals involving real and complex orbitals.

Of the distinct types of two-centre integrals that arise, six are of major 